\title{
Síntese e caracterização de beads de quitosana comercial reticulados com glutaraldeído
}

\author{
Synthesis and characterization of \\ commercial chitosan beads cross-linked \\ with glutaraldehyde
}

Maria Zillene Franklin da Silva Oliveira ${ }^{1}$, Tatiana Sainara Maia Fernandes ${ }^{2}$, Tecia Vieira Carvalho ${ }^{1,3}$

\footnotetext{
${ }^{1}$ Instituto Federal de Educação, Ciência e Tecnologia do Estado do Ceará, Campus Fortaleza, Departamento de Química e Meio Ambiente, av. Treze de Maio, 2081, CEP: 60040-531, Fortaleza, Ceará, Brasil.

${ }^{2}$ Universidade Federal do Ceará, Campus do Pici, Departamento de Química Analítica e Físico-Química, Rua Prof. Armando Farias, avenida Humberto Monte, Pici, Bloco 938/939, CEP: 60455-900, Fortaleza, Ceará, Brasil.

${ }^{3}$ Núcleo de Estudos e Pesquisas do Nordeste, Rua Felino Barroso, Bairro de Fátima, 643, CEP: 60050-130, Fortaleza, Ceará, Brasil.

e-mail: zillene26@gmail.com, tecia.carvalho@nepen.org.br, tatisainara@yahoo.com.br
}

\section{RESUMO}

A quitosana é um polímero natural com propriedades interessantes para remoção de compostos orgânicos. O presente trabalho trata da preparação e caracterização de beads de quitosana utilizando glutaraldeído (GLA) como agente reticulante, para serem futuramente serem aplicados na remoção de compostos orgânicos em matrizes aquosas. A variação na origem da quitosana ou nas suas condições de obtenção pode alterar suas propriedades físico-químicas, gerando modificações nas características dos beads, o que muitas vezes podem tornar os resultados não reprodutíveis.

Existe uma grande dificuldade em comparar resultados obtidos com os da literatura, pois ocorre uma grande quantidade de diferenças nas propriedades físico-químicas da quitosana. Assim, a determinação dessas propriedades ajuda a esclarecer as diferentes características encontradas para os beads, e que irão influenciar em sua aplicação. Inicialmente foi realizada a análise físico-química da quitosana comercial usada no estudo, visto que muitos fabricantes nacionais de quitosana não especificam a metodologia para sua obtenção, nem mesmo suas condições de processamento.

Os beads obtidos a partir de quitosana comercial foram preparados através de um sistema de gotejamento, após o processo de reticulação com glutaraldeído (GLA) $(0,75 \%$ 2,5\% e 25\%) as amostras foram caracterizadas por Espectroscopia de Infravermelho por Transformada de Fourier (FTIR), Fluorescência de Raios - X (FRX), Difração de Raios - X (DRX) e Microscopia Eletrônica de Varredura (MEV). O processo de reticulação promoveu alterações nas características de coloração dos beads, indicando efetivação da reticulação, que foi confirmada através da análise de DRX e MEV, visto que houve um aumento da cristalinidade. Com o FTIR foi possível visualizar as ligações formadas entre os grupos funcionais da quitosana com GLA, que ocorreu preferencialmente nos grupos amino. O preparo dos beads mostrou-se adequado, já que resultou em beads semelhantes ao encontrado na literatura.

Palavras-chave: Beads. Quitosana. Glutaraldeído. Caracterização.

\section{ABSTRACT}

Chitosan is a natural polymer with interesting properties for removing organic compounds. The present work deals with the preparation and characterization of chitosan beads using glutaraldehyde (GLA) as a crosslinking agent, to be applied in the future to remove organic compounds in aqueous matrices. The variation in the origin of the chitosan or in its conditions of obtaining can alter its physical-chemical properties, generating 
modifications in the characteristics of the beads, which often can make the results not reproducible.

There is a great difficulty in comparing the results obtained with those in the literature, as there are a large number of differences in the physical and chemical properties of chitosan. Thus, the determination of these properties helps to clarify the different characteristics found for the beads, and which will influence their application. Initially, the physical-chemical analysis of the commercial chitosan used in the study was performed, since many national chitosan manufacturers do not specify the methodology for obtaining it, not even its processing conditions.

The beads obtained from commercial chitosan were prepared using a drip system, after the crosslinking process with glutaraldehyde (GLA) $(0.75 \% 2.5 \%$ and $25 \%)$ the samples were characterized by Transformed Infrared Spectroscopy Fourier (FTIR), X-Ray Fluorescence (FRX), X-Ray Diffraction (DRX) and Scanning Electron Microscopy (SEM).

The crosslinking process promoted changes in the characteristics of the beads, changing their coloring characteristics, indicating the effectiveness of the crosslinking, which was confirmed through the XRD and SEM analysis, since there was an increase in crystallinity. With the FTIR it was possible to visualize the bonds formed between the functional groups of chitosan with GLA, which occurred preferentially in the amino groups. The preparation of the beads proved to be adequate, since it resulted in beads similar to that found in the literature.

Keywords: Beads. Chitosan. Glutaraldehyde. Characterization.

\section{INTRODUÇÃO}

Grandes quantidades de substâncias orgânicas são continuamente lançadas no ecossistema, onde o aumento da produção de efluentes orgânicos tem acompanhado o crescimento das diversas áreas da indústria [1]. Os componentes desses efluentes apresentam alta toxicidade, o que pode causar considerável dano ao meio ambiente. Por esse motivo, a pesquisa de materiais alternativos (resíduos industriais) para tratamento de águas residuais tem despertado um interesse crescente [2].

Biopolímeros como a quitosana, são uma alternativa sustentável, pois provêm de fontes renováveis, alta disponibilidade, baixo custo, e biodegradáveis, contemplando objetivos da química verde, e podendo ser usada em processos industrias que visam uma tecnologia mais limpa [2, 3]. A quitosana é um polímero biodegradável, biocompatível e atóxico, encontrado de modo natural em alguns fungos, e de modo mais comum, como um derivado do processo de desacetilação química da quitina, que consiste na transformação parcial dos grupos acetamina $\left(\mathrm{R}-\mathrm{NHCOCH}_{3}\right)$ da quitina em grupos amina $\left(\mathrm{R}-\mathrm{NH}_{2}\right)[4,5]$. O grande potencial da quitosana decorre da possibilidade de obtê-la em grande quantidade, podendo ser usada em diversas formas, como por exemplo, na forma de beads (esferas). [6]. A principal vantagem da quitosana é a existência de posições modificáveis em sua estrutura química, principalmente usando os grupos amina [7].

A quitosana é encontrada comercialmente em forma de pó. Ainda não existe um padrão internacional dos parâmetros físicos e químicos para este biopolímero. Por ser um polímero natural, a procedência da amostra e os tipos de tratamento que foram empregados para sua obtenção acabam por causar alterações no produto final modificando a massa molecular e viscosidade, resultando em diferentes amostras de quitosana, e ocasionando diferenças na síntese de beads. Por conta desses fatores, se faz necessária a caracterização deste biopolímero [8]. A caracterização da quitosana pode ser feita levando-se em consideração as propriedades físicas e químicas. As propriedades físicas incluem: tamanho das partículas, densidade, solubilidade e viscosidade. Já as propriedades químicas são a distribuição de massa molecular, grau de desacetilação, pH, índice de cristalinidade, valor de retenção de água, níveis de metais pesados e proteínas [9].

A modificação química da quitosana através da reticulação das cadeias do polímero ajuda a prevenir o inchaço, melhorar a seletividade para poluentes, aprimorar a sua resistência mecânica, bem como aumentar a sua estabilidade química em soluções ácidas e básicas. Todos esses recursos contribuem para sua alta eficiência de adsorção. A reticulação de quitosana com glutaraldeído (GLA) é um exemplo típico de modificação estrutural mediada por produto químico, com o intuito de evitar a dissolução do material reticulado em soluções ácidas ou para melhorar as suas propriedades de adsorção [10].

Assim, o objetivo desse trabalho foi realizar a análise físico-química de uma quitosana comercial, e depois produzir e caracterizar beads à base desta quitosana com reticulação com GLA para sua futura aplicação na remoção de compostos orgânicos em matrizes aquosas. 


\section{MATERIAIS E MÉTODOS}

\subsection{Identificação da Quitosana Comercial}

A quitosana foi obtida na Polymar (Brasil), sendo proveniente da carapaça do camarão. Algumas das características especificadas foram: nome químico (poli-2-deoxi-2-amino-glucose), natureza química (polímero linear combinado por ligações $\beta-(1-4)$-glicosídica), fórmula química $\left(\mathrm{C}_{6} \mathrm{H}_{11} \mathrm{O}_{4} \mathrm{~N}\right)_{\mathrm{n}}$, massa molecular (Monômero (161 Da); Polímero (60.000 - 300.000 Da) e método de fabricação (desacetilação da quitina).

\subsection{Análise Físico-Química da Quitosana Comercial}

No Quadro 1 são apresentados os parâmetros utilizados para caracterizar a quitosana comercial.

Quadro 1: Parâmetros fisico-químicos para caracterização da quitosana comercial.

\begin{tabular}{|l|l|c|}
\hline PARÂMETRO & FÓRMULA OU MÉTODO & REFERÊNCIA \\
\hline Grau de Desacetilação & $\% \mathrm{GD}=\left[\left(\mathrm{C}_{1} \mathrm{~V}_{1}-\mathrm{C}_{2} \mathrm{~V}_{2}\right) \times 161 \times 100\right] / \mathrm{G}$ & {$[11]$} \\
\hline $\mathrm{pH}$ & Potenciômetro & {$[12]$} \\
\hline Resíduos de Ignição & $\%$ Cinzas $=$ Peso inicial - Peso final $\times 100$ & {$[12]$} \\
\hline Perdas por dessecação & $\%=((\mathrm{P}-\mathrm{Q})) /(\mathrm{P} \times 100)$ & {$[12]$} \\
\hline Teor de Material Insolúvel & TMI $=$ Peso final $-\mathrm{P}$ inicial & {$[12]$} \\
\hline Viscosidade & Viscosímetro & {$[13]$} \\
\hline Peso Molecular & $\eta_{\mathrm{i}}=K \times\left(\mathrm{M}_{v}\right)^{a}$ & {$[14]$} \\
\hline
\end{tabular}

O GD é o grau médio de desacetilação em \%; $\mathrm{C}_{1}$ é concentração do $\mathrm{HCl}$ (mol/L); $\mathrm{V}_{1}$ é o volume de $\mathrm{HCl}(\mathrm{mL}) ; \mathrm{C}_{2}$ é a concentração do $\mathrm{NaOH}(\mathrm{mol} / \mathrm{L}) ; \mathrm{V}_{2}$ é o volume de $\mathrm{NaOH}(\mathrm{mL}) ; \mathrm{G}$ é o peso da amostra de quitosana (mg); 161 é a massa de uma unidade monomérica da quitosana; P é o peso inicial da quitosana (g); Q é o peso da quitosana seca $(\mathrm{g})$; $\eta_{\mathrm{i}}$ é a viscosidade intrínseca (obtida pelo viscosímetro); $\mathrm{M}_{v}$ é a massa molar viscosimétrica média; $K$ e $a$ são constantes que depende do polímero.

\subsection{Preparação dos Beads de Quitosana}

A quitosana foi dissolvida em solução de ácido acético a 2\%. A solução foi mantida em agitação por 12 a 24 horas até total solubilização do polímero. Após esse tempo, um funil de nylon foi usado para remover impurezas. Essa solução foi gotejada sobre uma solução de hidróxido de sódio $8 \%$ (p/v), com auxílio de um sistema de gotejamento (Figura 1) Os beads permaneceram na solução de hidróxido de sódio em agitação mecânica por 24h. Ao final, os beads foram lavados com água destilada até pH neutro, seguindo o procedimento descrito por FARIAS et al. [1].

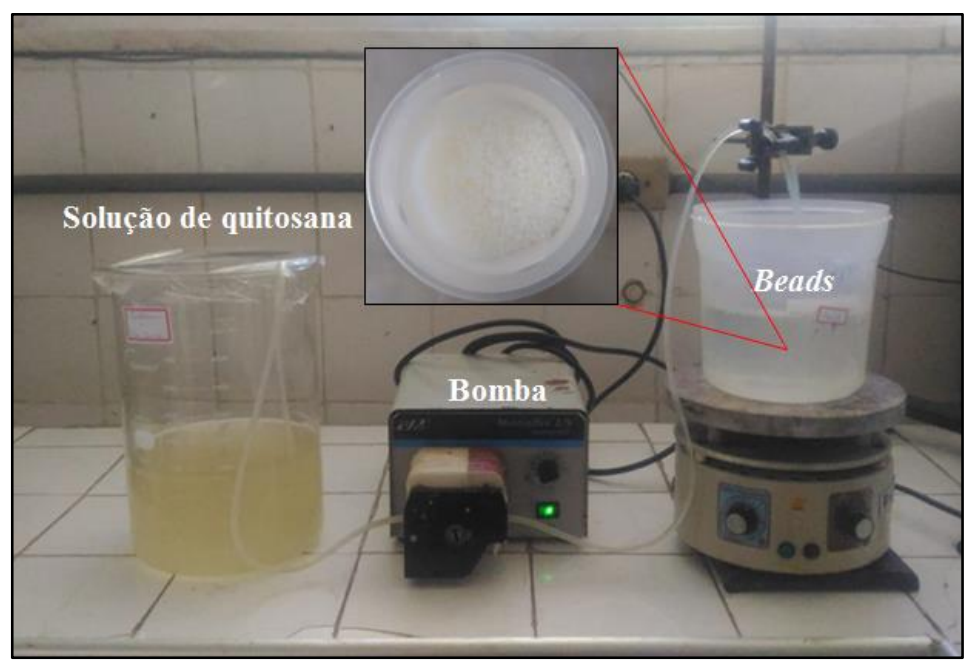

Figura 1: Sistema de gotejamento para produção dos beads. 


\subsection{Reticulação dos Beads}

Soluções aquosas de GLA nas concentrações de $0,75 \%$ 2,5\% e $25 \%$ foram usadas para o processo de reticulação, onde $5 \mathrm{~g}$ de beads úmidos de quitosana pura foram adicionados em $50 \mathrm{~mL}$ de cada uma destas três soluções de GLA, sem agitação, à temperatura ambiente por $2 \mathrm{~h}$. O procedimento foi adaptado de TORRES [15], NAING et al. [16] e FARIAS et al. [1].

\subsection{Avaliação Qualitativa}

A avaliação qualitativa foi executada através de observações visuais, levando-se em consideração a cor, a rigidez e a flexibilidade dos beads obtidos, além de se verificar a espessura dos mesmos.

\subsection{Caracterização}

\subsubsection{Espectroscopia de Infravermelho por Transformada de Fourier (FTIR)}

A fim de identificar a presença de grupos químicos funcionais específicos nos beads de quitosana pura e reticulados, foi utilizado um espectrômetro FTIR Perkin Elmer precisely, modelo Spectrum, usando pastilhas de $\mathrm{KBr}$ (brometo de potássio). As medidas foram realizadas no laboratório do Departamento de Química Orgânica e Inorgânica da Universidade Federal do Ceará (DQOI/UFC).

\subsubsection{Fluorescência de Raios - X (FRX)}

Foram realizadas análises de fluorescência de raios-X por dispersão de comprimento de onda com o intuito de identificar e determinar os elementos presentes nos biomateriais utilizados neste estudo. As análises foram realizadas utilizando um espectrômetro de Fluorescência de Raios-X por dispersão de comprimento de onda (modelo ZMS Mini II, Rigaku) pelo laboratório de Raios-X da Universidade Federal do Ceará.

\subsubsection{Difração de Raios - X (DRX)}

A verificação das fases cristalográficas foi realizada mediante medidas de difração de raios-X, conduzidas em difratômetro para amostras policristalinas modelo X-Pert PRO MPD-Panalytical, com spinner e geometria Bragg-Bretano operando em modo contínuo e radiação de $\mathrm{CuK} \alpha$ proveniente de tubo de cobre operado com foco linear a $40 \mathrm{kV}$ e $45 \mathrm{~mA}$. Os difratogramas foram obtidos no Laboratório de Raios-X da Universidade Federal do Ceará. A identificação de fases cristalinas foi realizada por meio do software X-Pert HighScore Plus (Panalytical).

\subsubsection{Microscopia Eletrônica de Varredura (MEV)}

As amostras de beads de quitosana com e sem reticulação foram analisados por microscopia eletrônica de varredura - MEV. Para observar a morfologia das superfícies das amostras foi utilizado um microscópio quanta FEG 450-FEI com magnificações de até 10.000 x, utilizando feixe de elétrons com aceleração de 20,0 $\mathrm{kV}$ e detectores de elétrons secundários e de energia dispersiva de raios-X no laboratório da Central Analítica da Universidade Federal do Ceará-UFC.

\section{RESULTADOS E DISCUSSÕES}

\subsection{Resultados da Análise Físico-Química}

As características físico-químicas da quitosana comercial estão apresentadas na Tabela 1. Este procedimento é necessário devido a muitos fabricantes nacionais de quitosana não especificarem a metodologia para sua obtenção, nem suas condições de processamento.

Para ARAÚJO [17] e BEZERRA [18] o grau de desacetilação é uma das características mais importantes da quitosana e pode variar entre 50 e $95 \%$ (a depender da metodologia utilizada). O valor encontrado foi de $70,6 \%$, o qual se encontra dentro da faixa relatada pelos autores supracitados. COSTA FILHO [19] destaca que quando o grau de desacetilação atinge $70 \%$ a quitosana é passível de ser solubilizada em soluções aquosas ácidas comportando-se como um polieletrólito catiônico, tornando-se possíveis variadas aplicações. 
Tabela 1: Parâmetros fisico-químicos para caracterização da quitosana comercial.

\begin{tabular}{l|c}
\hline PARÂMETRO & VALOR \\
\hline Grau de Desacetilação & $70,6 \%$ \\
\hline $\mathrm{pH}$ & 6,36 \\
\hline Resíduos de Ignição (cinzas) & $1,8 \%$ \\
\hline Perdas por dessecação & $11,1 \%$ \\
\hline Teor de Material Insolúvel & $0,96 \%$ \\
\hline Viscosidade & $161,2 \mathrm{cps}$ \\
\hline Peso Molecular & $268,87 \mathrm{kDa}$ \\
\hline
\end{tabular}

O teor residual de cinzas apresentou-se muito baixo, indicando que a quitosana utilizada nesse trabalho apresenta pureza elevada ( $<1 \%$ para amostras comerciais) [20]. ARAÚJO [17] obteve um teor de cinzas de $1,7 \%$ bem semelhante ao encontrado neste estudo. O nível de pureza da quitosana é um fator que tem um efeito profundo em sua solubilidade [21].

Geralmente, a quitosana é de natureza higroscópica e a quitosana comercial possui menos de $10 \%$ de teor de umidade [22]. A quitosana comercial apresentou um valor de umidade (perda por dessecação) de $11,1 \%$, sendo que ARAÚJO [17] encontrou um valor similar (11,5\%). O tempo de armazenamento é um dos fatores que pode aumentar o conteúdo de umidade da quitosana em até $20 \%$ [23]. Com um teor de água de $10 \%$, a quitosana contém uma molécula de água por unidade polimérica, onde nesse intervalo de concentração, as interações quitosana-água são altamente significativas [24]. A interação água-polímero influencia na estabilidade da quitosana, e consequentemente no preparo do material reticulado [25]. Quanto maior o conteúdo de água na estrutura da quitosana, mais rápido e pronunciado é o dano do polímero (via reações de hidrólise) e mais rápida é sua degradação [26].

A partir dos valores obtidos para viscosidade (161,1cps) e GD (70,6\%), ao observar os valores encontrados por SILVA [27], cuja viscosidade foi superior a $200 \mathrm{cps}$ e GD maior que $85 \%$, concluiu-se que de modo geral, a viscosidade aumenta com o grau de desacetilação. Tal autor afirma que a viscosidade de uma solução de quitosana aumenta à medida que aumenta a concentração da amostra, à medida que diminui a temperatura e aumenta o grau de desacetilação.

Por ser um biopolímero, qualquer variação na origem da matéria prima ou nas condições de obtenção altera as propriedades do produto final, o que muitas vezes tornam os resultados não reprodutíveis [14]. Desse modo, amostras distintas de quitosana podem apresentar massa molar variável. Segundo os critérios de WONG [28], a massa molar obtida neste estudo é classificada como sendo de média massa molar. LUPATINI [29] obteve uma massa molar (220,8 kDa) que corrobora com valores encontrado neste estudo (268, 87 $\mathrm{kDa}$ ), vale destacar que ambas as quitosanas são provenientes da carapaça do camarão.

\subsection{Resultados da Avaliação Qualitativa}

O Quadro 2 apresenta a nomenclatura das amostras, enquanto a Figura 2 mostra a comparação dos beads úmidos e secos, com seus respectivos diâmetros, na Figura 3 é feita a comparação do processo de liofilização dos beads.

Quadro 2: Nomenclatura dos beads de quitosana pura e reticulados.

\begin{tabular}{|l|l|l|}
\hline AMOSTRA & NOMENCLATURA & OBSERVAÇÕES \\
\hline Beads de quitosana pura & BQt & Branco, Rígido \\
\hline Beads de quitosana com $0,75 \%$ de glutaraldeído & BGLA1 & Marrom, Rígido \\
\hline Beads de quitosana com 2,5\% de glutaraldeído & BGLA2 & Marrom, Muito Rígido \\
\hline Beads de quitosana com $25 \%$ de glutaraldeído & BGLA3 & Marrom, Rígido \\
\hline
\end{tabular}




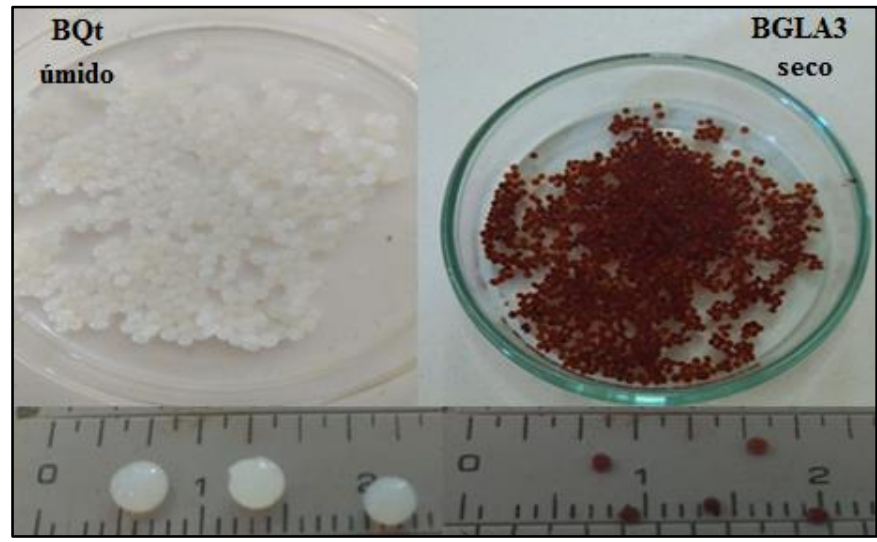

Figura 2: Beads úmidos e secos (temperatura ambiente) com seus respectivos diâmetros.

Com o processo de reticulação houve uma alteração da coloração que passaram de brancas para marrom. Os beads mostraram-se consistentes e uniformes, provavelmente devido ao eficiente controle do sistema de gotejamento. Em geral, os beads de BQt, BGLA1, BGLA2 e BGLA3 secos a temperatura ambiente apresentaram um diâmetro médio de $3,0 \mathrm{~mm}$, quando intumescidos. Após a evaporação do líquido os beads de todas as amostras é reduzido para 1,3 mm aproximadamente, ocorrendo quase 44\% de perda de diâmetro após a secagem. Geralmente beads úmidos possuem cerca de 90 - 95\% de conteúdo de água, denotando uma natureza hidrofílica [30]. Uma vez que a diminuição do tamanho dos beads foi resultado da perda de conteúdo de água, o resultado demonstra que os beads úmidos não possuem uma natureza tão hidrofílica. Beads expostos a maiores quantidades de GLA costumam apresentar maiores valores de massa molar, fazendo com que as matrizes formadas possuam uma estrutura mais rígida [31]. Houve diferença apenas na resistência dos beads, onde quanto mais GLA foi adicionado, mais frágeis os beads ficaram.

Com intuito de observar o aspecto dos beads após secagem, realizaram-se dois procedimentos, secagem a temperatura ambiente e liofilização (Figura 3). Concluiu-se que o modo de secagem influencia na característica final, uma vez que beads secos a temperatura ambiente mantiveram sua estrutura esférica e apresentaram-se bastante resistentes às forças físicas (Figura 2 - BGLA3 seco). Já os beads liofilizados apresentaram fragilidade e deformação na sua estrutura. Torres [15] destaca que bioadsorventes obtidos na forma úmida e que passam por processo de liofilização apresentam baixa resistência mecânica, podendo chegar ao rompimento dos poros.

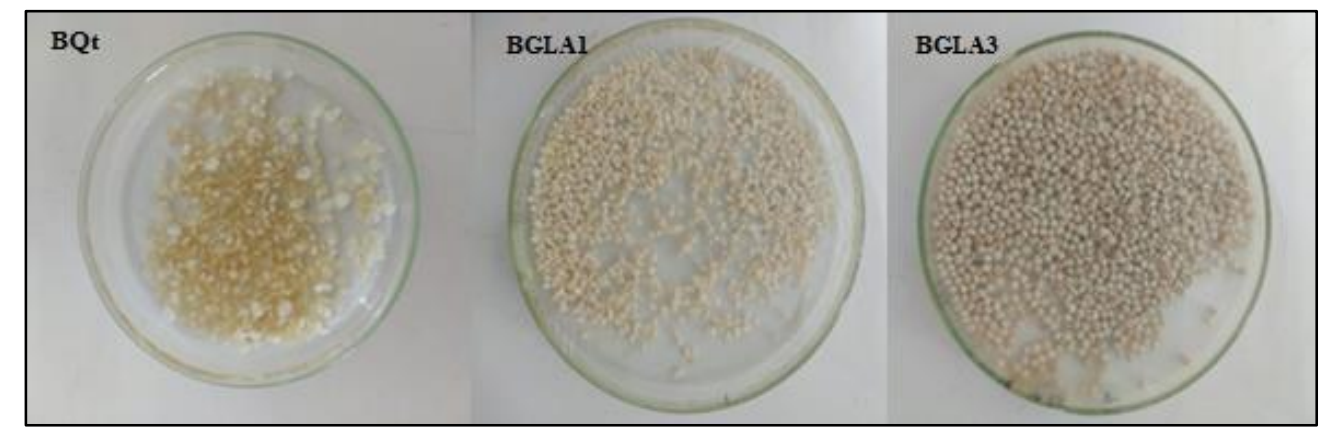

Figura 3: Beads liofilizados.

\subsection{Resultados da FTIR}

A Figura 4 mostra os espectros de absorção das amostras, que foram comparados para verificar a estrutura química da quitosana, e se houve modificação no processo de reticulação. Todas as amostras apresentam bandas características com estiramentos $\mathrm{O}-\mathrm{H}$ e N-H, entre $3000-3700 \mathrm{~cm}^{-1}$, estiramento $\mathrm{C}-\mathrm{H}$ (assimétrico) em $-\mathrm{CH}_{2}$ (grupos metílicos), entre 2923-2946 $\mathrm{cm}^{-1}$, onde tal banda é intensificada à medida que ocorre o aumento do GLA [32]. Nas amostras reticuladas, uma banda relacionada ao C-H (simétrico) aparece entre 2870-2892 $\mathrm{cm}^{-1}$, que também se intensifica com o aumento do GLA [33]. 


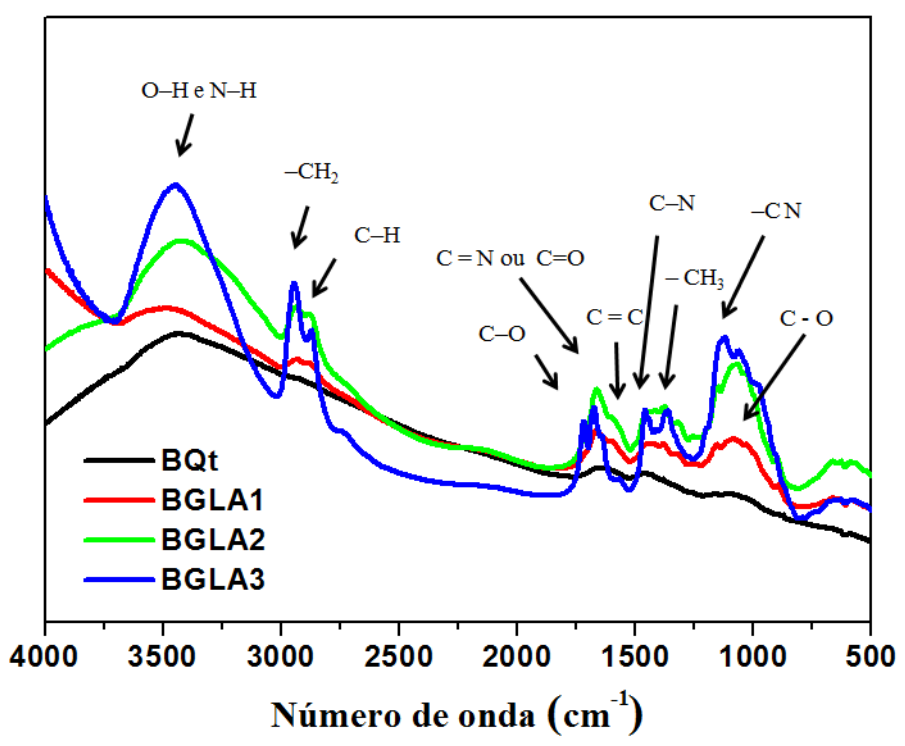

Figura 4: Espectros de FTIR para as amostras de beads.

A banda observada entre $1635-1678 \mathrm{~cm}^{-1}$ pode ser atribuída a deformação axial do grupo $\mathrm{C}=\mathrm{O}$ (amida) nos demais grupos acetila ou a ligação imina $(\mathrm{C}=\mathrm{N})$ [34]. A ligação $\mathrm{C}=\mathrm{N}$ é formada durante o processo de reticulação com GLA. A reticulação de quitosana com GLA se dá a partir da reação entre os grupos $-\mathrm{NH}_{2}$ e $-\mathrm{OH}$ do biopolímero com os grupos $-\mathrm{C}=\mathrm{O}$ do reticulante, culminando, dessa forma, na perda de uma molécula de água e na formação de ligação imina $(\mathrm{C}=\mathrm{N})$. O aparecimento de uma banda a $1700 \mathrm{~cm}^{-1}$ (BGLA2) e a $1718 \mathrm{~cm}^{-1}$ (BGLA3) pode estar relacionado com a presença de ligação de aldeído livre (grupos de aldeído que não reagiram) [1].

Nos beads reticulados, a banda entre $1568-1605 \mathrm{~cm}^{-1}$, indica a presença de ligação etilênica $(\mathrm{C}=\mathrm{C})$. $\mathrm{Na}$ banda entre 1455 a $1461 \mathrm{~cm}^{-1}$ é observada uma deformação do grupo amida $(\mathrm{C}-\mathrm{N})$. Outras deformações também são observadas entre as bandas $1362-1386 \mathrm{~cm}^{-1}$ e $1249-1264 \mathrm{~cm}^{-1}$, referentes ao grupo $-\mathrm{CH}_{3}$ [1]. As bandas entre 1075 e $1116 \mathrm{~cm}^{-1}$, correspondem à vibração do grupo amina [35]. Também ocorrem bandas na região de $668-661 \mathrm{~cm}^{-1}$ referentes ao surgimento de estruturas poliméricas que possuem anel aromático, fruto da reticulação dessa natureza [36].

\subsection{Resultados do FRX}

Na Tabela 2 são apresentados os elementos identificados através da análise de FRX para os beads. O cálcio foi o elemento que apresentou o teor mais pronunciado. A presença deste elemento tem relação direta com a etapa de desmineralização das carapaças de camarão, cujo objetivo é a remoção de minerais (principalmente $\mathrm{CaCO}_{3}$ ). A ineficiência do processo resulta na presença deste e demais minerais. A presença do cloro também é consequência da etapa de desmineralização uma vez que se utiliza ácido clorídrico $(\mathrm{HCl})$.

Tabela 2: FRX quantitativo dos beads (valores dados em \% de massa).

\begin{tabular}{c|c|c|c|c}
\hline ELEMENTOS & BQt & BGLA1 & BGLA2 & BGLA3 \\
\hline $\mathrm{Si}$ & 26,56 & - & - & 5,85 \\
\hline $\mathrm{S}$ & 1,08 & - & - & - \\
\hline $\mathrm{K}$ & 3,71 & 2,41 & - & 8,30 \\
\hline $\mathrm{Ca}$ & 47,01 & 57,84 & 62,50 & 73,08 \\
\hline $\mathrm{Fe}$ & 21,68 & 22,31 & 15,83 & - \\
\hline $\mathrm{Cl}$ & - & 2,45 & 6,70 & - \\
\hline $\mathrm{Cd}$ & - & 14,98 & - & - \\
\hline $\mathrm{P}$ & - & - & 5,85 & 14,51 \\
\hline $\mathrm{Br}$ & - & - & - & 4,11 \\
\hline
\end{tabular}


O Ferro e outros minerais (Tabela 2) são consequência dos reagentes alcalinos utilizados na neutralização convencional do $\mathrm{pH}$ da drenagem ácida $\left(\mathrm{CaO}, \mathrm{CaCO}_{3}, \mathrm{NaOH}, \mathrm{Na}_{2} \mathrm{CO}_{3}\right)$, que produzem um volume considerável de resíduos sólidos ricos em ferro e outros metais. A presença do cádmio e demais elementos é proveniente da contaminação da fonte natural da quitina e do processo de transformação da quitosana [37]. O próprio cultivo do camarão ou ração utilizada podem conter metais e causar bioacumulação no sistema digestório do camarão [38]. Por apresentar um grau de pureza de $25 \%$, o GLA pode ser uma possível fonte de contaminação, já que após a reticulação as concentrações de $\mathrm{P}, \mathrm{K}$ e $\mathrm{Cl}$ aumentaram.

\subsection{Resultados da DRX}

O DRX foi utilizado para investigar a mudança da estrutura da quitosana com e sem a reticulação. A Figura 5 apresenta os difratogramas das amostras dos beads. O difratograma de BQt apresentou dois picos, sendo um em torno de $2 \theta=12^{\circ}$ e outro por volta de $2 \theta=23,5^{\circ}$, evidenciando a parte cristalina do material, onde abaixo dos picos foi observada uma ampla faixa representando a forma amorfa do material.

O perfil semicristalino apresentado pela quitosana é decorrente das fortes interações inter e intramoleculares, o que confere certa organização à estrutura, sendo tais interações provenientes da presença de pontes de hidrogênio formadas entre os grupos amina, amida, álcool e outros grupos funcionais presentes na molécula [39]. No processo de reticulação ocorre a supressão dos dois picos de BQt anteriormente relatados, que desaparecem totalmente na maior concentração do agente reticulante (BGLA3).

O difratograma de BQt também apresenta um discreto pico em torno de $2 \theta=20,8^{\circ}$, cuja intensidade aumentou proporcionalmente em relação a concentração de GLA. Tal característica pode ser atribuída provavelmente a uma maior organização das cadeias da quitosana durante a reação de reticulação, ou pela orientação dos planos cristalinos durante o processo de formação do bead [40]. Verifica-se ainda picos de menor intensidade em $2 \theta=36,6^{\circ}, 42,5^{\circ}, 43^{\circ}$ e $48^{\circ}$, que ficaram também mais nítidos com o aumento da concentração do GLA. Yin et al. [41] relata que tais picos são característicos de regiões cristalinas da quitosana.
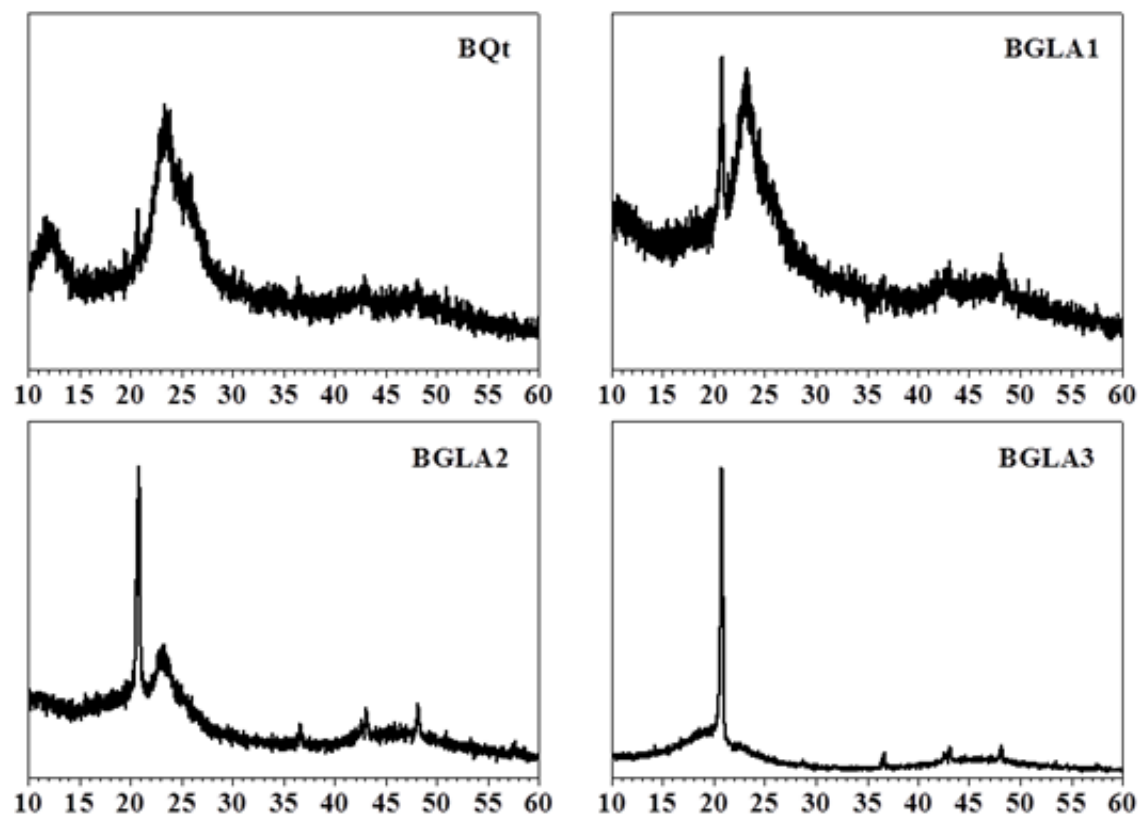

$2 \theta$ (graus)

Figura 5: Difratograma das amostras de beads.

\subsection{Resultados da MEV}

A análise de MEV foi realizada com a finalidade de se analisar a morfologia superficial dos adsorventes. A superfície de um material adsorvente possui grande importância na capacidade de remoção de um adsorvato, visto que, influencia no acesso das moléculas do soluto aos sítios de adsorção, facilitando ou dificultando este processo [7]. 
A Figura 6 mostra o MEV das amostras dos beads. O BQt apresenta falta de uniformidade na superfície, sem aparente estrutura de poros, onde segundo XUE e WILSON [42] isso é consistente com a natureza cristalina e devido as camadas desse polissacarídeo. Nos beads reticulados houve uma alteração significativa na superfície, tornando-a mais uniforme e com a presença de poros. Segundo Naing et al. [16] beads reticulados apresentam superfície mais áspera e com aspecto esponjoso do que beads não reticulados.

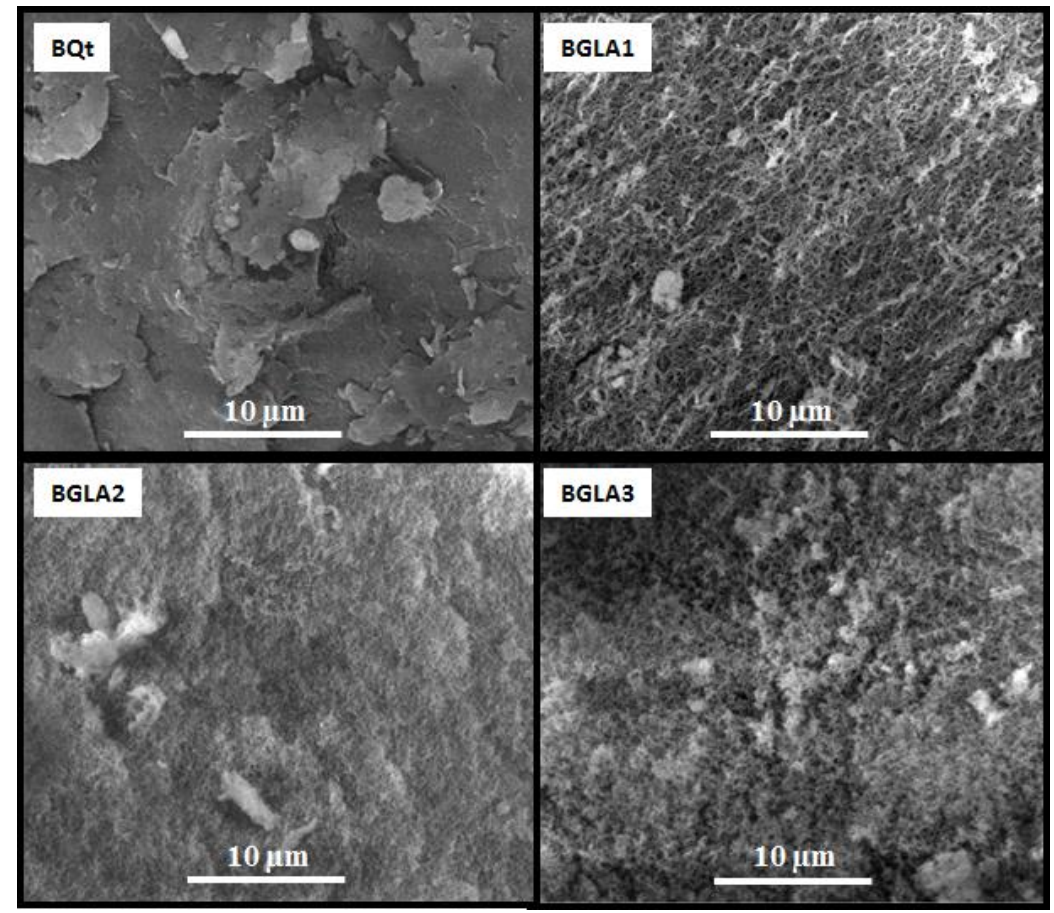

Figura 6: MEV das amostras de beads.

\section{CONCLUSÕES}

A determinação das propriedades físico-químicas da quitosana utilizada ajuda a esclarecer as diferentes características encontradas na literatura para os beads, e que irão influenciar em sua aplicação. O estudo da metodologia de preparação de beads de quitosana mostrou-se adequado. Os resultados da análise de FTIR indicaram que a reação de reticulação entre quitosana e GLA ocorre preferencialmente nos grupos amino e hidroxila, respectivamente. Os resultados de DRX e MEV mostraram que o GLA aumentou a cristalinidade e porosidade dos beads de quitosana, sendo estas características intensificadas com o aumento do grau de reticulação.

\section{AGRADECIMENTOS}

O presente trabalho foi realizado com apoio da CAPES - Coordenação de Aperfeiçoamento de Pessoal de Nível Superior, bem como o Laboratório de Análises de Traços - LAT e Parque de Desenvolvimento Tecnológico - PADETEC.

\section{BIBLIOGRAFIA}

[1] FARIAS, P.V.S., ARAGÃO, D.C., FARIAS, M.V., et al., "Natural and cross-linked chitosan spheres as adsorbents for diesel oil removal”, Adsorption Science \& Technology, v. 33, n. 9, pp. 783-792, 2015.

[2] VIDAL, R.R.L., MORAES, J.S., "Removal of organic pollutants from wastewater using chitosan: a literature review", International Journal of Environmental Science and Technology, v. 16, pp. 1741-1754, 2018.

[3] CIDREIRA, J.O., MACÊDO, R.R., RANGEL, F.C., et al., "Catalisadores ácidos baseados na simples modificação da quitosana para a esterificação do ácido oleico". Revista Matéria, v. 23, n. 4, 2018.

[4] KANMANI, P., ARAVIND, J., KAMARAJ, M., et al., "Environmental applications of chitosan and cellulosic biopolymers: a comprehensive outlook", Bioresource Technology, v. 242, pp. 295-303, Out. 2017. 
[5] NAZ, F., ZUBER, M., ZIA, K.M., et al., "Synthesis and characterization of chitosan-based waterborne polyurethane for textile finishes", Carbohydrate Polymers, v. 200, pp. 54-62, 2018.

[6] BEDADE, D.K., SUTAR, Y.B., SINGHAL, R.S. "Chitosan coated calcium alginate beads for covalent immobilization of acrylamidase: process parameters and removal of acrylamide from coffee", Food Chemistry, v. 275, pp. 95-104, 2019.

[7] SIGNINI, R., MOREIRA, J.V.I., DE SOUSA, R.B., et al., "Avaliação da adsorção de íons níquel (II) e cádmio (II) em quitosana, quitosana reticulada e carboximetilquitosana reticulada", Revista Virtual de Química, v.11, n. 1, pp. $237-254,2019$.

[8] SANTOS, J.E.D., SOARES, J.D.P., DOCKAL, E.R., et al., "Caracterização de quitosanas comerciais de diferentes origens", Polímeros, v. 13, n. 4, pp. 242-249, 2003.

[9] DE QUEIROZ ANTONINO, R.S.C.M., LIA FOOK, B.R.P., DE OLIVEIRA LIMA, V.A., et al., "Preparation and characterization of chitosan obtained from shells of shrimp (Litopenaeus vannamei Boone)", $M a-$ rine Drugs, v. 15, n. 5, 141, 2017.

[10] MARQUES, J.S., PEREIRA, M.R., SOTTO, A., et al., "Removal of aqueous copper (II) by using crosslinked chitosan films", Reactive and Functional Polymers, v. 134, pp. 31-39, 2019.

[11] DOMARD, A., RINAUDO, M., "Preparation and characterization of fully deacetylated chitosan", International Journal of Biological Macromolecules, v. 5, n. 1, pp. 49-52, 1983.

[12] UNITED STATES PHARMACOPEIA USP 23, pp. 1746, 1801, 1819, 1995.

[13] BARROS, F.C., CAVALCANTE, R.M., CARVALHO, T.V., et al., "Produção e caracterização de esfera de quitosana modificada quimicamente". Revista Iberoamericana de Polímeros, v. 7, n. 4, pp. 232-246, 2006.

[14] FONSECA, A.C.M., Processos de obtenção e caracterização físico-química de quitinas e quitosanas extraídas dos rejeitos da indústria pesqueira da região de Cananéia-SP. Dissertação de M.Sc., Instituto de Pesquisas Energéticas e Nucleares, São Paulo, SP, 2016.

[15] TORRES, M.A., VIEIRA R.S., BEPPU, M.M., et al., "Produção e Caracterização de Microesferas de Quitosana Modificadas Quimicamente", Polímeros, v.15, n. 4, pp. 306-312, 2005.

[16] NAING, N.N., LI, S.F.Y., LEE, H.K., "Application of porous membrane-protected chitosan microspheres to determine benzene, toluene, ethylbenzene, xylenes and styrene in water", Journal of Chromatography $A$, v. 1448, pp. 42-48, 2016.

[17] ARAÚJO, D.O., Quitosana como suporte para imobilização de aspergillus nigeran 400 visando biodegradação de águas residuárias contaminadas com metil paration, Dissertação de M.Sc., Instituto Federal de Educação, Ciência e Tecnologia do Ceará - Campus Fortaleza, Fortaleza, CE, 2015.

[18] BEZERRA, A.M., Síntese e avaliações físico-químicas e biológicas de derivados de quitosana de alta e baixa massa molecular, Dissertação de M.Sc., Universidade de São Paulo, São Paulo, SP, 2011.

[19] COSTA FILHO, C.F.B., Produção de quitosana a partir de exoesqueleto de camarão (Litopenaeus vannamei) em diferentes tamanhos de partículas avaliando a atividade antibacteriana em bactérias gram positivas e gram negativas, Dissertação de M.Sc., Universidade Católica de Pernambuco, Recife, PE, 2017.

[20] TOLAIMATE, A., DESBRIERES, J., RHAZI, M., et al., "Contribution to the prepa-ration of chitins and chitosans with controlled physico-chemical properties”, Polymers, v. 44, n. 26, pp. 7939-7952, 2003.

[21] SZYMAŃSKA, E., WINNICKA, K., "Stability of chitosan-a challenge for pharmaceutical and biomedical applications", Marine Drugs, v. 13, n. 4, pp. 1819-1846, 2015.

[22] ANAND, M., KALAIVANI, R., MARUTHUPANDY, M., et al., "Extraction and characterization of chitosan from marine crab and squilla collected from the Gulf of Mannar Region, South India", Journal of Chitin and Chitosan Science, v. 2, n. 4, 280-287, 2014.

[23] NO, H.K., PRINYAWIWATKUL, W., "Stability of chitosan powder during long-term storage at room temperature", Journal of Agricultural and Food Chemistry, v. 57, n. 18, pp. 8434-8438, 2009.

[24] NETO, C.D.T., GIACOMETTI, J.A., JOB, A.E., et al., "Thermal analysis of chitosan based networks", Carbohydrate Polymers, v. 62, n. 2, pp. 97-103, 2005. 
[25] CAPITANI, D., CRESCENZI, V., DE ANGELIS, A.A., et al., "Water in hydrogels. An NMR study of water/polymer interactions in weakly cross-linked chitosan networks", Macromolecules, v. 34, n. 2, pp. 4136-4144, 2001.

[26] VILJOEN, J.M., STEENEKAMP, J.H., MARAIS, A.F., et al., "Effect of moisture content, temperature and exposure time on the physical stability of chitosan powder and tablets", Drug Development and Industrial Pharmacy, v. 40, n. 6, pp. 730-742, 2014.

[27] SILVA, J.N., Esferas de alumina funcionalizadas com nanopartículas de ouro e prata obtidos a partir do biopolímero quitosana, Dissertação de M.Sc., Universidade Federal de Pernambuco, Recife, PE, 2010.

[28] WONG, T.W., "Chitosan and its uses in desing of insulin delivery system", Recents Patents on Drug Delivery \& Formulation, v. 3, n. 1, pp. 8 - 25, 2009.

[29] LUPATINI, K.N., Desenvolvimento de membranas de quitosana para aplicação em células a combustível, Dissertação de M.Sc., Universidade Estadual do Oeste do Paraná, Cascavel, PR, 2016.

[30] CONTRERAS-CORTÉS, A.G., ALMENDARIZ-TAPIA, F.J., GÓMEZ-ÁLVAREZ, A., et al., “Toxicological assessment of cross-linked beads of chitosan-alginate and Aspergillus australensis biomass, with efficiency as biosorbent for copper removal", Polymers, v. 11, n. 2, pp. 222 (2-17), 2019.

[31] KULKARNI, A.R., SOPPIMATH, K.S., AMINABHAVI, T.M., et al., "Glutaraldehyde crosslinked sodium alginate beads containing liquid pesticide for soil application”, Journal of Controlled Release, v. 63, n. 1-2, pp. 97-105, 2000.

[32] WU, C.H., et al., "Improvement of oxygen release from calcium peroxide - polyvinyl alcohol beads by adding low - cost bamboo biochar and its applicationin bioremediation", Clean Soil Air Water, v. 43, n. 2, pp. $287-295,2014$.

[33] COSTA Jr., E.S., MANSUR, H.S. "Preparação e caracterização de blendas de quitosana/poli(álcool vinílico) reticuladas quimicamente com glutaraldeído para aplicação em engenharia de tecido", Química Nova, v. 31, n. 6, pp. $1460-1466,2008$.

[34] CONSTANTINI, S.M., et al., "Formulation and caracterization of new polymeric Systems based on chitosan and xanthine derivates with thiazolidin -4- one scaffold”, Materials, v. 12, n. 4, pp. 558, 2019.

[35] BEPPU, M.M., et al., "Síntese e caracterização de estruturas densas e porosas de quitosana", Polímeros, v. 9 , n. 4 , pp. $163-169,1999$.

[36] FARIA, C.C., TONELLO, P.S., "Estudo comparativo de adsorção de íons metálicos em meio aquoso por membranas de quitosana reticuladas", Revista Brasileira de Ciências Ambientais, n. 47, pp. 101-115, 2018.

[37] TAOKAEW, S., et al., "Size distribution and characteris tics of chitin microgels prepard via emulsified reverse - micelle", Materials, v. 12, n. 7, pp. 1160, 2019.

[38] CAVALHEIRO, T.B., et al., "Crescimento do camarão litopenaeus vannamei em viveiros e tanques utilizando efluente do processo de dessalinização”, Gaia Scientia, v. 10, n. 4, pp. 319 -337, 2016.

[39] UrAGAMI T., TOKURA S., Material Science of Chitin and Chitosan., $1^{\mathrm{a}}$ ed. Kodansha, Ltd. Springer, 2006.

[40] SILVA, M.C., et al., "Esferas de quitosana/curcumina pelo método de gelificação ionotrópica: influência da incorporação do fármaco", Revista Eletrônica de Materiais e Processos, v. 10, n. 1, pp. 21-28, 2015.

[41] YIN, X., et al., "Metal-coordinating controlled oxidative degradation of chitosan and antioxidant activity of chitosan-metal complex”, ARKIVOC, v. 9, pp. 66-78, Ago. 2004.

[42] XUE, C., WILSON, L.D, "Kinetic study on urea uptake with chitosan based materials", Carbohydrate Polymers, v. 135, pp. 180 - 186, 2016.

\section{ORCID}

Maria Zillene Franklin da Silva Oliveira

Tatiana Sainara Maia Fernandes

Tecia Vieira Carvalho https://orcid.org/0000-0002-9157-9251

http://orcid.org/0000-0003-2961-5124

https://orcid.org/0000-0001-9999-5009 